\title{
Knockdown of collagen $\alpha-1$ (III) inhibits glioma cell proliferation and migration and is regulated by miR128-3p
}

\author{
YUAN-FENG GAO ${ }^{1,2}$, TAO ZHU ${ }^{2}$, JUAN CHEN ${ }^{2}$, LIN LIU $^{1}$ and RONG OUYANG ${ }^{1}$ \\ ${ }^{1}$ Department of Pharmacy, The First Hospital of Hunan University of Chinese Medicine, \\ Changsha, Hunan 410007; ${ }^{2}$ Department of Clinical Pharmacology, Xiangya Hospital, \\ Central South University, Changsha, Hunan 410008, P.R. China
}

Received January 13, 2018; Accepted May 22, 2018

DOI: $10.3892 / \mathrm{ol} .2018 .8830$

\begin{abstract}
As a member of the collagen family, collagen $\alpha-1$ (III) (COL3A1) is an important protein in the development and progression of several tumors. However, the role of COL3A1 in glioma is not yet clear. The present study examined the expression and function of COL3A1 in glioma cell behavior and identified microRNA (miRNA) regulators. It was demonstrated that COL3A1 expression was upregulated in glioma and directly correlated with the tumor grade. Analysis of the GSE4290 and GSE7696 profiles acquired from the Gene Expression Omnibus database also revealed an increased COL3A1 expression in malignant gliomas compared with the lower grade gliomas and non-tumor brain tissue, which was directly correlated with glioma grade. To explore the functional role of COL3A1 in glioma cell growth, small interfering RNA interference was applied to inhibit COL3A1 expression in Hs683 and U251 cells. The relative COL3A1 mRNA and protein expression levels were significantly reduced in the knockdown cells as determined by western blot analysis. In addition, decreased COL3A1 expression in Hs683 and U251 glioma cells resulted in a delay in cell growth and colony disruption as determined by MTS and colony formation assays. Wound healing analysis indicated that cells with suppressed expression of COL3A1 had a reduced ability to migrate. COL3A1 mRNA levels were inversely correlated with the miR128-3p level in glioma, suggesting that miR128-3p expression is associated with COL3A1 inhibition as verified by reverse transcription-quantified polymerase chain reaction. These results suggest that COL3A1 may be a novel regulator of glioblastoma cell behavior and may represent a novel target for gene therapies against glioma.
\end{abstract}

Correspondence to: Professor Rong Ouyang, Department of Pharmacy, The First Hospital of Hunan University of Chinese Medicine, 95 ShaoShan Road, Changsha, Hunan 410007, P.R. China E-mail: ouyangrong126@126.com

Key words: glioma, collagen $\alpha-1(\mathrm{III})$, proliferation, migration, microRNA128-3p

\section{Introduction}

Glioma is the most common form of central nervous system (CNS) neoplasm. This tumor is mostly expanded or infiltrated, and has no clear boundary with normal brain tissues. Therefore, it has a high degree of malignancy and poor prognosis (1). In addition, according to the World Health Organization (WHO) glioma grading criteria, gliomas can be classified as low-grade (WHO I-II) and high-grade (WHO III-IV) according to their degree of malignancy (2). Despite considerable advances in surgical resection (or a biopsy if surgery cannot be performed), radiotherapy and chemotherapy, the prognosis for glioma has not significantly improved $(2,3)$. Median survival time of patients with glioblastoma multiforme, the most common and malignant type of glioma, is only 14.6 months, and the 5-year survival rate is less than $10 \%$ (4-6). In order to solve the problem of glioma recurrence after surgical resection, gene therapy against glioma has become a hot topic. Therefore, identification of tumor biomarkers as target genes is essential for developing targeted therapy against glioma (7). One promising family for gene targeting is the collagen family. According to the distribution in the body, collagen can be divided into interstitial collagen, basement membrane collagen and peripheral collagen. So far, 29 types of collagen have been identified and described. A typical collagen molecule, also called the triple helix, is made up of three left hand spirals (proline II), intertwined and bonded to each other, forming a long and tough right hand spiral structure (8-10).

COL3A1, also known as collagen $\alpha-1(\mathrm{III})$, is a precursor of collagen III- $\alpha$ protein found in extensible connective tissues such as skin, lung, and vascular system, and frequently in association with type I collagen (COL1A1). An increase of COL3A1 and COL1A1 is found in some cancers, resulting as markers of poor prognosis $(11,12)$. COL3A1 is a fibrillary collagen molecule that has been linked to myocardial infarction (13) and increased risk of stroke recurrence and prognosis in Chinese patients (14). Interestingly, COL3A1 is the target of miR29 family and downregulation of these microRNA (miRNA) family members is responsible for the increased invasiveness of nasopharyngeal carcinoma (15). Let-7d suppresses growth, metastasis, and tumor macrophage infiltration by targeting COL3A1 and CCL7 in renal cell carcinoma (16). Additionally, an integrated computational analysis of mutations regarding 
miRNA and mRNA expression in a TCGA dataset of glioblastoma multiforme patients revealed interesting insights into collagen regulation in glioma (17). In our previous study, we also revealed that COL3A1 and SNAP91 may serve as suitable biomarkers for diagnostic or therapeutic strategies against glioma by using bioinformatics (18). The current research on COL3A1 is only in bioinformatics analysis. However, the role of COL3A1 in tumorigenesis and progression, particularly in glioma, is still limited.

Therefore, in this study, we examined the role of COL3A1 in glioma tumorigenesis and progression. Our results showed that COL3A1 expression was upregulated and directly correlated with glioma grade. Using COL3A1-siRNA, we discovered that COL3A1 expression silencing resulted in an inhibition of cell proliferation and migration. Additionally, COL3A1 mRNA levels were inversely correlated with miR128-3p level in glioma. These findings identify COL3A1 as an oncogene in glioma that could potentially serve as a therapeutic target for glioma treatment.

\section{Materials and methods}

Cell culture and transient transfection. Human glioma cell lines U87, U251, U343 and Hs683 were obtained from the Institute of Biochemistry and Cell Biology of the Chinese Academy of Sciences (Shanghai, China) and maintained as adherent cultures in Dulbecco's modified Eagle's medium (DMEM C11995500BT; Gibco; Thermo Fisher Scientific, Inc., Waltham, MA, USA) supplemented with $10 \%$ fetal bovine serum (FBS, 10099-141; Gibco; Thermo Fisher Scientific, Inc.). Cells were cultured at $37^{\circ} \mathrm{C}$ in a humidified atmosphere of $5 \% \mathrm{CO}_{2}$. Cells were detached using $0.25 \%$ trypsin when they were approximately $80 \%$ confluent and consequently sub-cultured.

A COL3A1-siRNA and negative control siRNA (NC-siRNA) were purchased from Santa-Cruz Biotechnology, Inc. (Dallas, TX, USA (sc-43062; sc-637007). miRNA mimics and negative control miRNA were purchased from Guangzhou RiboBio Co., Ltd. (Guangzhou, China). Cells were transfected using Lipofectamine ${ }^{\circledR}$ RNAiMAX reagent (13778150; Invitrogen; Thermo Fisher Scientific, Inc.) according to the manufacturer's protocol.

Patient samples and quantitative real-time PCR (qPCR). The consecutive newly diagnosed 33 glioma patients included in this study were recruited from the Affiliated Cancer Hospital of XiangYa School of Medicine, Central South University (Changsha, Hunan, China) and informed consent was obtained from all patients prior to the beginning of the study (CTXY-1300041-3). Samples were collected after surgical resection, snap-frozen in liquid nitrogen and stored at $-80^{\circ} \mathrm{C}$ before RNA extraction. Total RNA was extracted by trizol reagent according to the manufacturer's protocol. Two micrograms of RNA were reverse-transcribed into cDNA using the Primescript RT reagent kit with gDNA Eraser (Takara Bio Inc., Otsu, Japan). To increase the output of the microRNAs, RNA was reverse-transcribed into cDNA using Mir- $\mathrm{X}^{\mathrm{TM}}$ miRNA FirstStrand Synthesis kits (Clontech Laboratories, Inc., Mountainview, CA, USA). qPCR was performed using SYBR Premix DimerEraser kit (Takara Bio Inc.). qPCR conditions were the following: $95^{\circ} \mathrm{C}$ for $30 \mathrm{sec}, 40$ cycles at $95^{\circ} \mathrm{C}$ for $5 \mathrm{sec}, 55^{\circ} \mathrm{C}$ for $30 \mathrm{sec}$ and $72^{\circ} \mathrm{C}$ for $30 \mathrm{sec}$. Primers used for qPCR were as follows: COL3A1 forward, TTGAAGGAG GATGTTCCCATCT; reverse, ACAGACACATATTTGGCA TGGTT. GAPDH forward, GAGTCAACGGATTTGGTC GT; reverse, TTGATTTTGGAGGGATCTCG. U6 forward, CTCGCTTCGGCAGCACA; reverse, AACGCTTCACGA ATTTGCGT. Mature miRNA was used as miRNA specific 5' primer. The 3' primer for qPCR was supplied with the kit. All the analyses were performed in duplicate. The relative expression of COL3A1 mRNA/microRNA was normalized to the expression level of GAPDH/U6 mRNA using the $2^{-\mathrm{ACq}}$ method (19).

Immunohistochemistry. Paraffin embedded glioma tissues were cut into $5 \mu \mathrm{m}$ sections and stained to evaluate COL3A1 expression. The whole process was performed according to standard protocols. The primary antibody (HPA007583; Sigma-Aldrich, St. Louis, MO, USA) was diluted to 1:100 and incubated overnight at $4^{\circ} \mathrm{C}$. Color development was performed by using the DAB kit (P0203; Beyotime Biotechnology, Shanghai, China) according to the manufacturer's protocol. Two pathologists evaluated the immunohistochemistry results independently, according to the percentage of COL3A1 positive cells per field of vision at $\mathrm{x} 400$ magnification. Positive cell rate was measured by Image pro-plus 6.0 software.

Western blot analysis. Total cell protein extracts were obtained using RIPA buffer purchased from Beyotime Biotechnology. Protein lysates were separated by centrifugation, and the concentrations were qualified using Pierce BCA Protein assay kit (P0012; Beyotime Biotechnology). Protein lysates were loaded into a $10 \%$ SDS-PAGE gel. The gel was transferred to a $0.45 \mu \mathrm{m}$ PVDF membrane (Millipore, Billerica, MA, USA) for $2 \mathrm{~h}$. Primary antibodies anti-COL3A1 (1:1,000, HPA007583; Sigma-Aldrich), and anti-GAPDH (1:5,000, G8795; Sigma-Aldrich) were incubated overnight at $4^{\circ} \mathrm{C}$. Secondary HRP-conjugated antibodies (A0208/A0216; Beyotime Biotechnology) were added at 1:2,000 dilution and incubated for $1 \mathrm{~h}$ at $25^{\circ} \mathrm{C}$. The bound antibodies were visualized using an enhanced chemiluminescence reagent (RPN2232; GE Healthcare Life Sciences, Little Chalfont, UK) and quantified by densitometry using ChemiDoc XRS+ image analyzer (Bio-Rad Laboratories, Inc., Hercules, CA, USA). Densitometric band analyses were adjusted to the GAPDH loading control.

[3-(4,5-dimethylthiazol-2-yl)-5-(3-carboxymethoxyphenyl)-2(4-sulfophenyl)-2H-tetrazolium] MTS assay. Hs683 and U251 were seeded onto 96 -well culture plates $(1,000$ cells/well/100 $\mu \mathrm{l})$ and cell proliferation was measured at $24,48,72$, and $96 \mathrm{~h}$ after transfection using a MTS reagent (1:9; Promega Corporation, Madison, WI, USA) according to the manufacturer's instructions. Absorbance was measured at $490 \mathrm{~nm}$ by the BioTek ${ }^{\circledR}$ Eon (Synergy ${ }^{\mathrm{TM}}$ HT; BioTek Instruments, Inc., Winooski, VT, USA). Background absorbance of the medium was subtracted.

Colony formation assay. U251 were transfected with siRNA for $48 \mathrm{~h}$ and were collected and seeded in triplicate into 6-well 

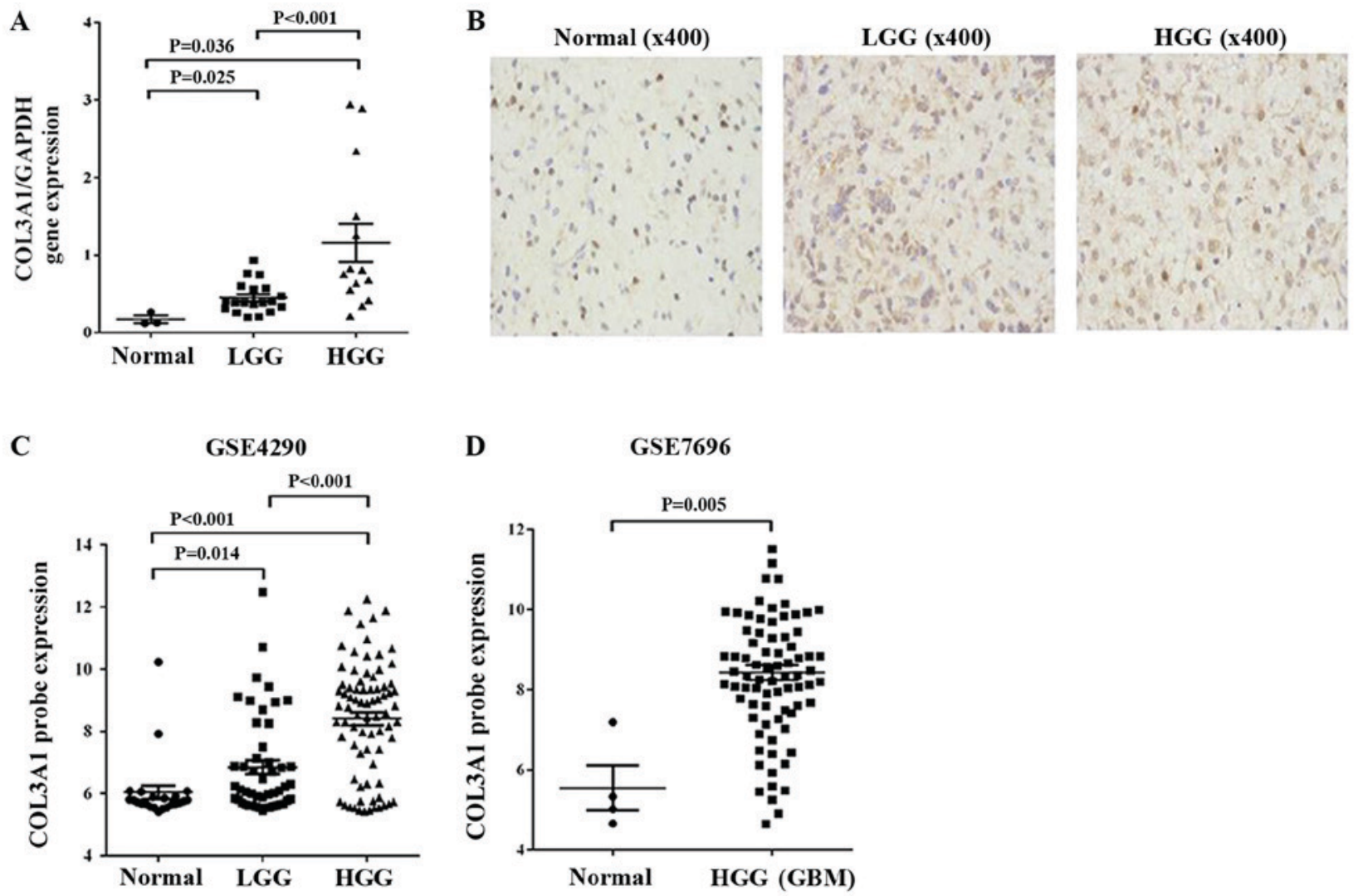

Figure 1. COL3A1 was significantly upregulated in glioma. (A) COL3A1 was upregulated and directly correlated with the glioma grade in 33 glioma samples. (B) COL3A1 protein expression showed the same results as above. Magnification, $\mathrm{x} 400$. Data analysis also showed that COL3A1 expression was upregulated in glioma both in (C) GSE4290 and (D) GSE7696 datasets. One-way ANOVA followed by Tukey's post hoc test. COL3A1, collagen $\alpha-1$ (III); LGG, low grade glioma; HGG, high grade glioma; GBM, glioblastoma multiforme; PR, positive cell rate.

plates at a density of 1,000 cells/well $/ \mathrm{ml}$. Cells were incubated for 10 days at $37^{\circ} \mathrm{C}$ under $5 \% \mathrm{CO}_{2}$ atmosphere. Cells were then fixed with $4 \%$ paraformaldehyde for $30 \mathrm{~min}$ and stained with Giemsa (C0121; Beyotime Biotechnology) for $20 \mathrm{~min}$. After washing with double-distilled $\mathrm{H}_{2} \mathrm{O}$ several times, images from the cell plates were obtained using a camera (Canon, Inc., Tokyo, Japan).

Wound healing assay. U251 were seeded onto a 6-well plate overnight. The confluent monolayers were scratched using sterile pipette tips and washed with phosphate-buffered saline (PBS) several times to remove detached cells. Cells were then transfected with siRNA for $48 \mathrm{~h}$ in medium without serum. Photographs of the wounded areas were obtained using a Leica DMI3000 B inverted microscope (Leica Microsystems $\mathrm{GmbH}$, Wetzlar, Germany). The migration rate was calculated according to the scratched surfaces, which were quantified using ImageJ Version 1.41o software (National Institutes of Health, Bethesda, MD, USA).

Bioinformatics analysis. Three online miRNA research databases, TargetScan (http://www.targetscan.org), microRNA. org (http://34.236.212.39/microrna/home.do), and miRBase (http://www.mirbase.org/), were used to predict miRNA regulators of the COL3A1 gene. The predicted miRNAs were selected based on the coincident results of the three databases. Overlapping results were extracted (excluding those that have been studied) for subsequent analysis.
Dual luciferase activity assay. U251 cells were seeded onto a 24-well plate overnight. Cells were co-transfected with pmiR-RB-reporter vectors (WT/MUT) and miRNA mimics (Guangzhou RiboBio Co., Ltd.) using Lipofectamine 2000 (Invitrogen; Thermo Fisher Scientific, Inc.). After 48 h, luciferase activity was measured using the Dual Luciferase Reporter assay system (Promega). Firefly luciferase activity was normalized to renilla luciferase activity for each transfected well. The results were obtained from three independent experiments and each one was performed in triplicate.

Statistical analysis. SPSS 19.0 software was used for statistical analyses. The significance of the differences two groups was estimated by Student's t-test. One-way ANOVA followed by Tukey's post hoc test was used to analyze differences between three or more groups. The association between COL3A1 and clinical features was analyzed using Chi-square test. Pearson correlation analyses were performed to investigate the correlation between COL3A1 and miR128-3p. All tests performed were two-sided and statistical significance was set at $\mathrm{P}<0.05$.

\section{Results}

COL3A1 is strongly upregulated in glioma. To evaluate if COL3A1 is upregulated in glioma and directly correlated with glioma grade, we examined COL3A1 in 33 glioma tissues and normal brain tissues using quantitative qPCR. COL3A1 expression was significantly higher in glioma tissues [low grade 
Table I. Correlation between COL3A1 expression and glioma clinicopathologic features in 33 patients.

\begin{tabular}{|c|c|c|c|c|c|}
\hline \multirow[b]{2}{*}{ Characteristic } & \multirow[b]{2}{*}{$\mathrm{N}(\%)$} & \multicolumn{2}{|c|}{$\begin{array}{c}\text { COL3A1 } \\
\text { expression levels }\end{array}$} & \multirow[b]{2}{*}{ Ratio (high/low) } & \multirow[b]{2}{*}{ P-value } \\
\hline & & High & Low & & \\
\hline \multicolumn{6}{|l|}{$\operatorname{Sex}$} \\
\hline Male & $12(36.36)$ & 4 & 8 & 0.500 & 0.462 \\
\hline Female & $21(63.64)$ & 5 & 16 & 0.312 & \\
\hline \multicolumn{6}{|l|}{ Age, years } \\
\hline$<45$ & $16(48.49)$ & 5 & 11 & 0.454 & 0.257 \\
\hline$\geq 45$ & $17(51.51)$ & 4 & 13 & 0.307 & \\
\hline \multicolumn{6}{|l|}{ Grade } \\
\hline Low (I+II) & $19(57.57)$ & 3 & 16 & 0.187 & 0.0008 \\
\hline High (III+IV) & $14(42.43)$ & 6 & 8 & 0.750 & \\
\hline
\end{tabular}

The tissue samples were divided into two groups based on the median expression level.
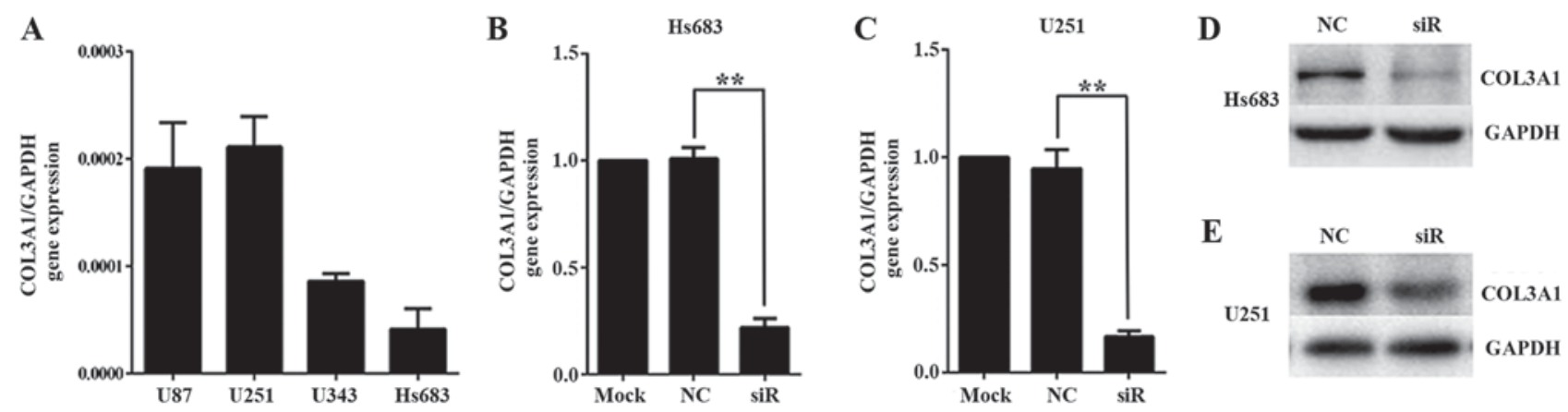

Figure 2. SiRNA inhibited COL3A1 expression efficiently in human glioma cell lines. (A) COL3A1 was higher in U87 and U251, and the lowest in Hs683 by reverse transcription-quantitative polymerase chain reaction. Transfection with COL3A1-siRNA led to a $\sim 80 \%$ reduction in the expression of COL3A1 mRNA in (B) Hs683 and (C) U251 and evident depletion of COL3A1 protein expression in (D) Hs683 and (E) U251. One-way ANOVA followed by Tukey's post hoc test. ${ }^{* *} \mathrm{P}<0.01$. si, short interfering; NC, negative control; COL3A1, collagen $\alpha-1(\mathrm{III})$.

glioma (LGG), $\mathrm{P}=0.025$; high grade glioma (HGG), $\mathrm{P}=0.036$ ] compared with normal brain samples (Fig. 1A). Thirty-three glioma tissues were divided into two group, based on the median expression level of all gliomas. Chi-square test was performed to analyze the data shown in Table I. As summarized, COL3A1 was significantly associated with WHO grade (LGG vs. HGG, $\mathrm{P}=0.0008$ ). Further examination of COL3A1 protein expression (positive cell rate) resulted in 'normal brain' (7.71\%), LGG (18.58\%), HGG (24.03\%), separately (Fig. 1B). In our previous study, we used TCGA data to verify previous COL3A1 expression. Here we evaluated its expression in GSE4290 and GSE7696 expression profiles acquired from the Gene Expression Omnibus (GEO, http://www.ncbi.nlm.nih.gov/ geo/) database. Upregulated COL3A1 (>2-fold upregulated) expression was observed in malignant gliomas compared to LGG and non-tumor brain tissue and resulted directly correlated with the glioma grade in GSE4290 dataset (Fig. 1C). Analysis of the GSE7696 data also revealed a >2-fold COL3A1 upregulation at the transcription level and resulted drastically increased in malignant gliomas when compared to non-tumor brain tissue (Fig. 1D). These results suggested a correlation between increased COL3A1 expression and glioma tumor grade.
SiRNA inhibits COL3A1 expression efficiently in human glioma cell lines. To investigate the role of COL3A1 in glioma, we knocked down COL3A1 expression using a COL3A1-siRNA (Fig. 2). COL3A1 gene expression was higher in U87 and U251, and the lowest in Hs683 (Fig. 2A). After transfection of Hs683 and U251 with COL3A1-siRNA or NC-siRNA, COL3A1 mRNA expression was significantly reduced by $\sim 80 \%$ and protein expression in Hs683 (Fig. 2B and D) and U251 (Fig. 2C and E) was nearly depleted. Therefore, the present results demonstrated that a highly efficient COL3A1 knockdown at the mRNA and protein level was achieved after transfection.

COL3A1 knockdown suppresses proliferation and migration in glioma cells. To investigate the effect of COL3A1 silencing on glioma cell proliferation, Hs683 and U251 transfected with COL3A1-siRNA or NC-siRNA were analyzed by MTS assay. Proliferation of Hs683 cells treated with COL3A1-siRNA began to decrease $72 \mathrm{~h}$ after transfection. On day 4, proliferation of silenced cells was significantly reduced compared to those treated with NC-siRNA or untreated cells $(\mathrm{P}<0.05$; Fig. 3A). These results were consistent with the results obtained 
A

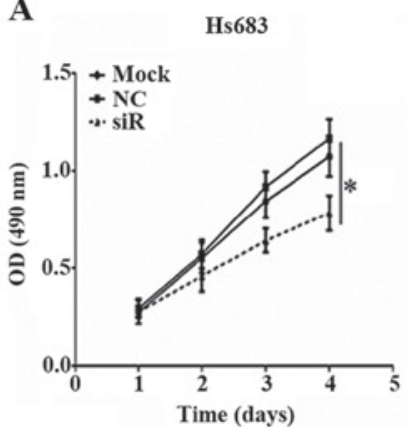

B

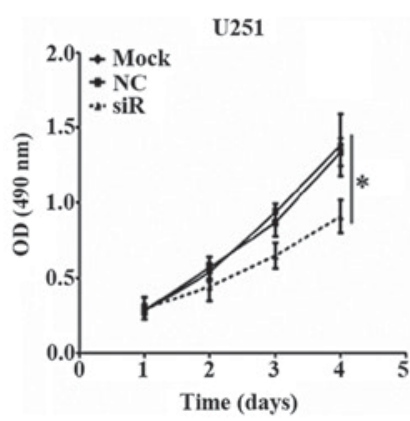

C

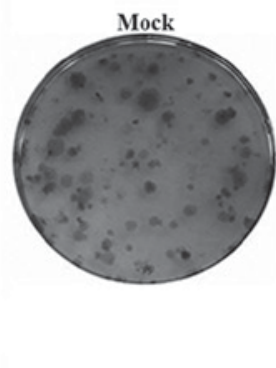

E

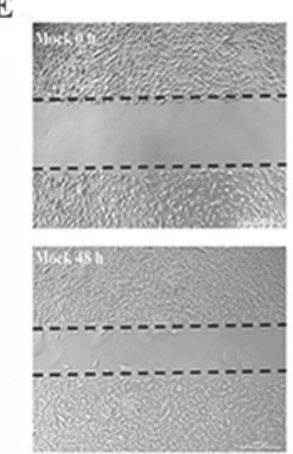

U251 (x1)
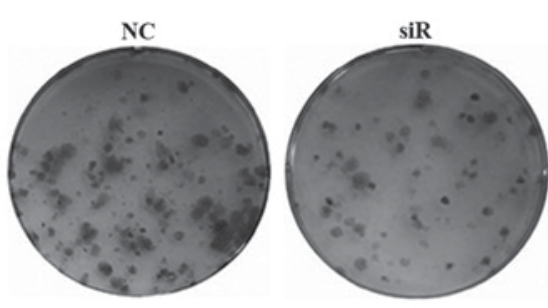

$\mathbf{U} 251(\mathbf{x 1 0 0})$
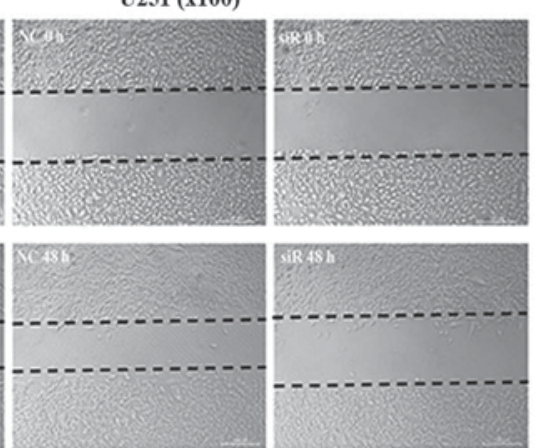

D

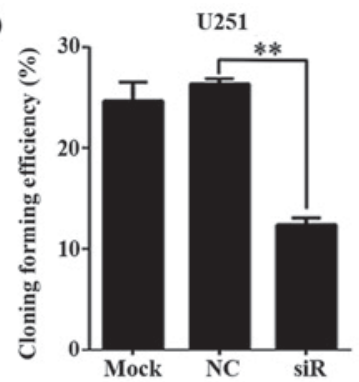

F
U251

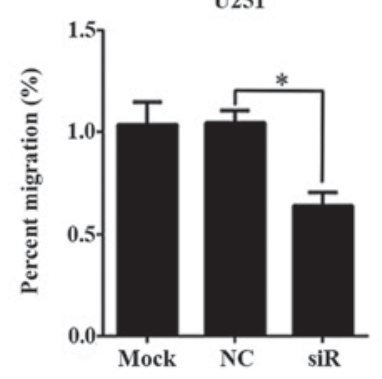

Figure 3. COL3A1 knockdown suppressed proliferation in glioma cells. MTS assay. The absorbance in COL3A1-siRNA group was remarkably reduced in

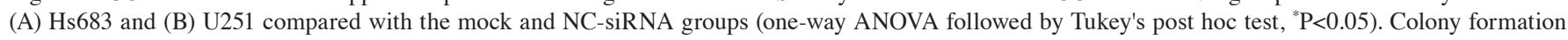
assay. (C and D) COL3A1 silencing in U251 cells resulted in a substantial reduction in colony formation (one-way ANOVA followed by Tukey's post hoc test, ${ }^{* *} \mathrm{P}<0.01$ ); wound healing assay. (E) COL3A1 silencing dramatically reduced glioma cell migration in U251 cells (magnification, $\mathrm{x} 100$ ). (F) Migration rates were significantly lower in COL3A1-siRNA treated cells compared with non-treated and NC-siRNA treated cells. One-way ANOVA followed by Tukey's post hoc test, ${ }^{*} \mathrm{P}<0.05$. COL3A1, collagen $\alpha-1(\mathrm{III})$; si, short interfering; ANOVA, analysis of variance; NC, negative control.

in U251 cells $(\mathrm{P}<0.05$; Fig. 3B). Moreover, no significant difference was observed between NC-siRNA treated cells and untreated cells. The relatively long-term effects of COL3A1 knockdown on glioma cell proliferation were also examined using a colony formation assay. As shown in Fig. 3C and D, COL3A1 silencing in U251 cells substantially reduced colony formation $(\mathrm{P}<0.01)$.

Next, we investigated the effect of COL3A1 silencing on cell migration. COL3A1 knockdown dramatically reduced glioma cell migration $48 \mathrm{~h}$ after transfection with COL3A1-siRNA (Fig. 3E). Migration rates were significantly lower in silenced cells compared to untreated and NC-siRNA treated cells $(\mathrm{P}<0.05$; Fig. 3F). No significant differences were found between untreated and NC-siRNA treated cells. These results suggested that COL $3 \mathrm{~A} 1$ contributed to glioma cell migration. Taken together, MTS, Colony formation assay and Wound healing assay were used to illustrate the relationship between COL3A1 and cell proliferation in Fig. 3. These findings identify COL3A1 as an oncogene in glioma.

miR128-3p overexpression inhibits COL3A1 mRNA and protein expression. To identify potential miRNA regulators of the COL3A1 gene, three online miRNA research databases, TargetScan, microRNA.org, and miRBase, were analyzed using internetwork. Among the predicted miRNAs, we were particularly interested in miR128-3p because of our previous results indicating this miRNA as associated with COL3A1. To validate miR128-3p binding, we identified a putative consensus site for miR128-3p binding the 3UTR of COL3A1 using microRNA.org. Furthermore, luciferase activity decreased significantly in the cells transfected with the wild-type reporter plasmids but not in those transfected with mutant miR128-3p plasmids in U251 cells ( $\mathrm{P}<0.05$; Fig. 4A). Moreover, COL3A1 mRNA was inhibited by miR128-3p overexpression $(\mathrm{P}<0.05)$, while miR128-3p mRNA showed no significant differences when transfection with COL3A1-siRNA was performed in U251 cells (Fig. 4B). COL3A1 protein was also inhibited by the miR128-3p mimics in U251 cells (Fig. 4C). Additionally, COL3A1 mRNA levels were inversely correlated with miR128-3p level in glioma tissues (Fig. 4D).

\section{Discussion}

Gliomas is one of the most common malignant tumors with high recurrence, fatality and low recovery rates. Glioma cells are highly invasiveness and infiltrative multiple brain regions (20-23). Targeting specific tumor-promoting genes may provide a promising alternative treatment to the current standards (24). Collagen is a biological macromolecular protein synthetized and widely present in animal cells, which content and sort is various. It has a significant impact on cells, tissues and organs. Previously, we identified COL3A1 and SNAP91 as potential biomarkers for diagnostic or therapeutic strategies against glioma using bioinformatics (18). In the current study, we confirmed that COL3A1 was upregulated in glioma by analyzing COL $3 \mathrm{~A} 1$ expression in 33 glioma samples and two datasets (GSE4412, GSE7696). Additionally, silencing COL3A1 expression in vitro inhibited glioma cell proliferation and migration. This might be closely related to the following three primary functions of collagen in brain tumors: to 
A

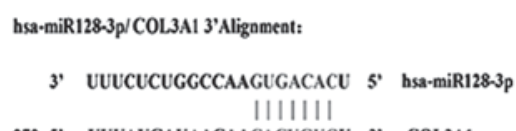

272: 5' uUdatgauacaicacugugu 3 ' col3a1

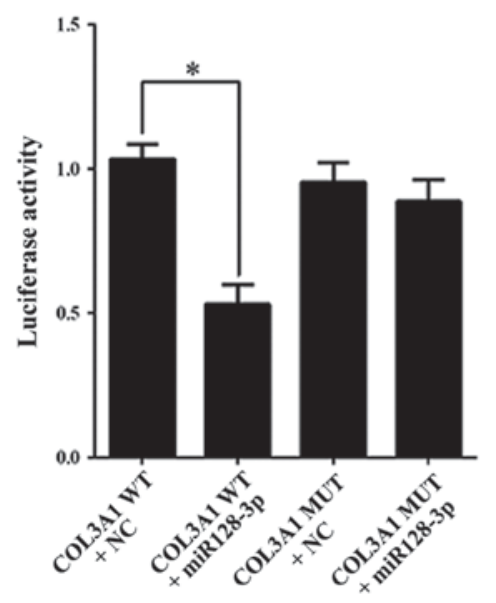

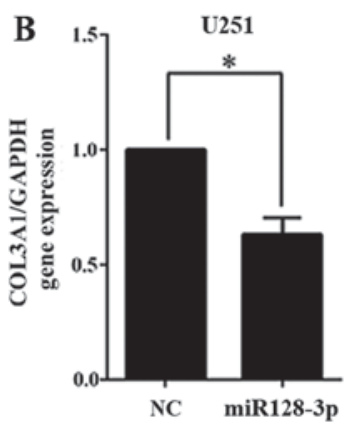

D

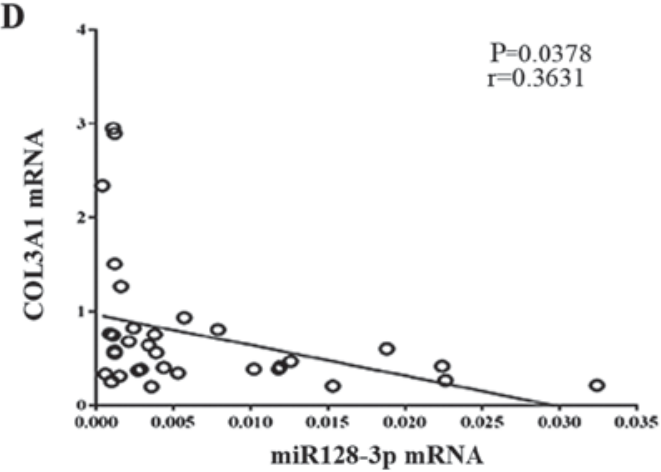

$\mathbf{U} 251$

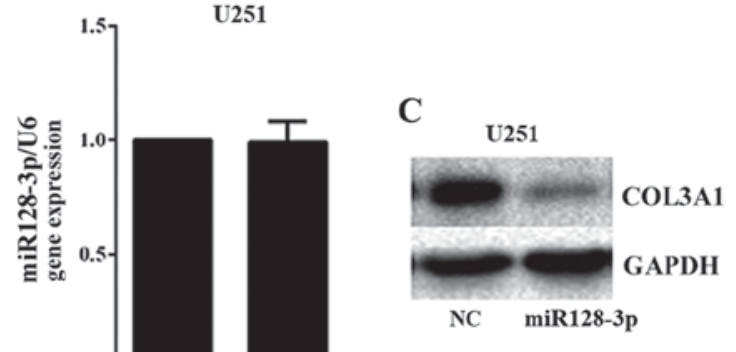

Figure 4. COL3A1 mRNA and protein expression was inhibited by miR-128 overexpression. (A) Dual luciferase activity assay. Luciferase activity decreased significantly in the cells transfected with the wild-type reporter plasmids but not in those transfected with mutant miR128-3p plasmids in U251 cells (one-way ANOVA followed by Tukey's post hoc test, $\left.{ }^{*} \mathrm{P}<0.05\right)$. (B) COL3A1 was inhibited by miR128-3p overexpression (Student's t-test, $\left.{ }^{*} \mathrm{P}<0.05\right)$, while miR128-3p showed no significant differences when transfected with COL3A1-siRNA in U251 cells. (C) COL3A1 protein expression was also inhibited by the miR128-3p mimics in U251 cells. (D) COL3A1 gene expression was inversely correlated with miR128-3p level in glioma tissues. miR, microRNA; COL3A1, collagen $\alpha-1(\mathrm{III})$; si, short interfering; ANOVA, analysis of variance; NC, negative control.

act as: i) a scaffold and provide sites for cell adhesion; ii) a reservoir for extracellular matrix proteins, proteoglycans and growth factors; and iii) a ligand to activate signal transduction networks required for tumor growth, differentiation and invasion (25-29). Although the underlying mechanism for these effects still needs to be studied, our results gave a hint on the role of COL3A1 in collagen and brain tumor. Taken together, the results suggested that COL3A1 functions as an oncogene in glioma.

Interestingly, COL3A1 is the target of the miR29 family members, and downregulation of these members is responsible for the increased invasiveness of nasopharyngeal carcinoma (15). Boxing Su et al validated COL3A1 as a direct, functional target gene for let-7d in renal cell carcinoma, in vitro and in vivo (16). Our results also found that the 3'UTRs in COL3A1 were the target sites through which miR128-3p modulates the expressions of COL3A1. Importantly, COL3A1 mRNA was inversely correlated with miR128-3p levels in glioma clinical specimens. Our findings suggested that miR128-3p-COL3A1 regulatory pathway plays a role in glioma growth. These findings indicated COL3A1 as an oncogene in glioma that could potentially serve as a target in the therapy for glioma patients.

Since COL3A1 is involved in the invasion of nasopharyngeal carcinoma progression (15), invasion studies should be conducted. In addition, any candidate miRNA, which may involve the COL3A1 should be taken into consideration. Unfortunately, because of the financial strain, in the current research there is a lack of data on miR128-3p expression in glioma tissue and invasion studies. We mainly discussed the role of COL3A1 in glioma. We have done the MTS test with U87 and the results was consistent with the trend of U251 and Hs683 (data not shown). Although U87 cells (that matches U87 MG ATCC cells) have been reported to be misidentified (30), we confirm that such misidentification issue is unlikely to affect the outcomes of our study. In addition, due to the lack of patient's prognostic information, survival analysis could not be performed. For this reason, we will continue to collect data regarding the patient's prognostic information. Then, future studies should be performed in glioma cells on other COL3A1 miRNA regulators reported in other tumors.

Although glioma gene therapy is beneficial for patients, it is still under test and did not achieve the desired effect in clinical trials. As a result, an improvement of gene therapy clinical application to ameliorate the effect and the development of more efficient methods of using gene therapy is the focus of our future research. Our goal is a continuous gene therapy innovation to provide more scientific and feasible strategies for the clinical treatment of glioma.

\section{Acknowledgements}

Not applicable.

\section{Funding}

This study was supported by the Key specialty of clinical pharmacy of Hunan administration of traditional Chinese medicine (grant no. 2017-11), the Education Bureau of Hunan 
Province (grant no. 17A156) and the Hunan administration of traditional Chinese medicine (grant no. 201709).

\section{Availability of data and materials}

The datasets used and/or analyzed during the current study are available from the corresponding author on reasonable request.

\section{Authors' contributions}

RO and YG conceived the project, designed experiments, analyzed data, and wrote the manuscript. YG, TZ, JC, and LL performed experiments and analyzed data.

\section{Ethics approval and consent to participate}

All glioma human samples were taken in accordance with the ethical guidelines of the Affiliated Cancer Hospital of XiangYa School of Medicine, Central South University. The present study was approved by the Ethics Committee of Central South Unviersity and written informed consent was obtained from all patients prior to their inclusion within the present study.

\section{Consent for publication}

All patients agreed to the publication of their data.

\section{Competing interests}

The authors declare that they have no competing interests.

\section{References}

1. Dodgshun AJ, Maixner WJ, Heath JA, Sullivan MJ and Hansford JR: Single agent carboplatin for pediatric low-grade glioma: A retrospective analysis shows equivalent efficacy to multiagent chemotherapy. Int J Cancer 138: 481-488, 2016.

2. Gao YF, Zhu T, Mao CX, Liu ZX, Wang ZB, Mao XY, Li L, Yin JY, Zhou HH and Liu ZQ: PPIC, EMP3 and CHI3L1 are novel prognostic markers for high grade glioma. Int J Mol Sci 17: pii: E1808, 2016.

3. Karsy M, Guan J, Sivakumar W, Neil JA, Schmidt MH and Mahan MA: The genetic basis of intradural spinal tumors and its impact on clinical treatment. Neurosurg Focus 39: E3, 2015.

4. Batista A, Riedemann L, Vardam T and Jain RK: Targeting the tumor microenvironment to enhance pediatric brain cancer treatment. Cancer J 21: 307-313, 2015.

5. Domingues P, González-Tablas M, Otero Á, Pascual D, Miranda D, Ruiz L, Sousa P, Ciudad J, Gonçalves JM, Lopes MC, et al: Tumor infiltrating immune cells in gliomas and meningiomas. Brain Behav Immun 53: 1-15, 2016.

6. Panditharatna E, Yaeger K, Kilburn LB, Packer RJ and Nazarian J: Clinicopathology of diffuse intrinsic pontine glioma and its redefined genomic and epigenomic landscape. Cancer Genet 208: 367-373, 2015.

7. Tobias A, Ahmed A, Moon KS and Lesniak MS: The art of gene therapy for glioma: A review of the challenging road to the bedside. J Neurol Neurosurg Psychiatry 84: 213-322, 2013.

8. Dodd A and Daniels TR: Injectable recombinant human platelet-derived growth factor in collagen carrier for hindfoot fusion. Foot Ankle Clin 21: 777-791, 2016.
9. Huang S, Vader D, Wang Z, Stemmer-Rachamimov A, Weitz DA, Dai G, Rosen BR and Deisboeck TS: Using magnetic resonance microscopy to study the growth dynamics of a glioma spheroid in collagen I: A case study. BMC Med Imaging 8: 3, 2008.

10. Liang Y, Diehn M, Bollen AW, Israel MA and Gupta N: Type I collagen is overexpressed in medulloblastoma as a component of tumor microenvironment. J Neurooncol 86: 133-141, 2008.

11. Zhang Z, Wang Y, Zhang J, Zhong J and Yang R: COL1A1 promotes metastasis in colorectal cancer by regulating the WNT/ PCP pathway. Mol Med Rep 17: 5037-5042, 2018.

12. Wang XQ, Tang ZX, Yu D, Cui SJ, Jiang YH, Zhang Q, Wang J, Yang PY and Liu F: Epithelial but not stromal expression of collagen alpha-1(III) is a diagnostic and prognostic indicator of colorectal carcinoma. Oncotarget 7: 8823-8838, 2016.

13. Kong CH, Lin XY, Woo CC, Wong HC, Lee CN, Richards AM and Sorokin VA: Characteristics of aortic wall extracellular matrix in patients with acute myocardial infarction: tissue microarray detection of collagen I, collagen III and elastin levels. Interact Cardiovasc Thorac Surg 16: 11-15, 2013.

14. Lv W, Lin Y, Song W, Sun K, Yu H, Zhang Y, Zhang C, Li L, Suo M, Hui R and Chen J: Variants of COL3A1 are associated with the risk of stroke recurrence and prognosis in the Chinese population: A prospective study. J Mol Neurosci 53: 196-203, 2014.

15. Qiu F, Sun R, Deng N, Guo T, Cao Y, Yu Y, Wang X, Zou B, Zhang S, Jing T, et al: miR-29a/b enhances cell migration and invasion in nasopharyngeal carcinoma progression by regulating SPARC and COL3A1 gene expression. PLoS One 10: e0120969, 2015.

16. Su B, Zhao W, Shi B, Zhang Z, Yu X, Xie F, Guo Z, Zhang X, Liu J, Shen Q, et al: Let-7d suppresses growth, metastasis, and tumor macrophage infiltration in renal cell carcinoma by targeting COL3A1 and CCL7. Mol Cancer 13: 206, 2014.

17. Dong H, Luo L, Hong S, Siu H, Xiao Y, Jin L, Chen R and Xiong M: Integrated analysis of mutations, miRNA and mRNA expression in glioblastoma. BMC Syst Biol 4: 163, 2010.

18. Gao YF, Mao XY, Zhu T, Mao CX, Liu ZX, Wang ZB, Li L, Li X, Yin JY, Zhang W, et al: COL3A1 and SNAP91: Novel glioblastoma markers with diagnostic and prognostic value. Oncotarget 7: 70494-70503, 2016.

19. Livak KJ and Schmittgen TD: Analysis of relative gene expression data using real-time quantitative PCR and the 2(-Delta Delta C(T)) method. Methods 25: 402-408, 2001.

20. Xue S, Song G and Yu J: The prognostic significance of PD-L1 expression in patients with glioma: A meta-analysis. Sci Rep 7: 4231, 2017.

21. Xue J, Zhao Z, Zhang L, Xue L, Shen S, Wen Y, Wei Z, Wang L, Kong L, Sun H, et al: Neutrophil-mediated anticancer drug delivery for suppression of postoperative malignant glioma recurrence. Nat Nanotechnol 12: 692-700, 2017.

22. Wang P and Heitman J: The cyclophilins. Genome Biol 6: 226 , 2005.

23. Qi XT, Zhan JS, Xiao LM, Li L, Xu HX, Fu ZB, Zhang YH, Zhang J, Jia XH, Ge G, et al: The unwanted cell migration in the brain: Glioma metastasis. Neurochem Res 42: 1847-1863, 2017.

24. Fan YC, Cui CC, Zhu YS, Zhang L, Shi M, Yu JS, Bai J and Zheng JN: Overexpression of CAP1 and its significance in tumor cell proliferation, migration and invasion in glioma. Oncol Rep 36: 1619-1625, 2016.

25. Lucey P and Goldberg DJ: Complications of collagen fillers. Facial Plast Surg 30: 615-622, 2014.

26. Raglow $\mathrm{Z}$ and Thomas SM: Tumor matrix protein collagen XI $\alpha 1$ in cancer. Cancer Lett 357: 448-453, 2015.

27. Konstantopoulos A and Mehta JS: Conventional versus accelerated collagen cross-linking for keratoconus. Eye Contact Lens 41: 65-71, 2015.

28. Jokerst C, Purdy $\mathrm{H}$ and Bhalla S: An overview of collagen vascular disease-associated interstitial lung disease. Semin Roentgenol 50: 31-39, 2015.

29. Snedeker JG and Gautieri A: The role of collagen crosslinks in ageing and diabetes-the good, the bad, and the ugly. Muscles Ligaments Tendons J 4: 303-308, 2014.

30. Allen M, Bjerke M, Edlund H, Nelander S and Westermark B: Origin of the U87MG glioma cell line: Good news and bad news. Sci Transl Med 8: 354re3, 2016. 